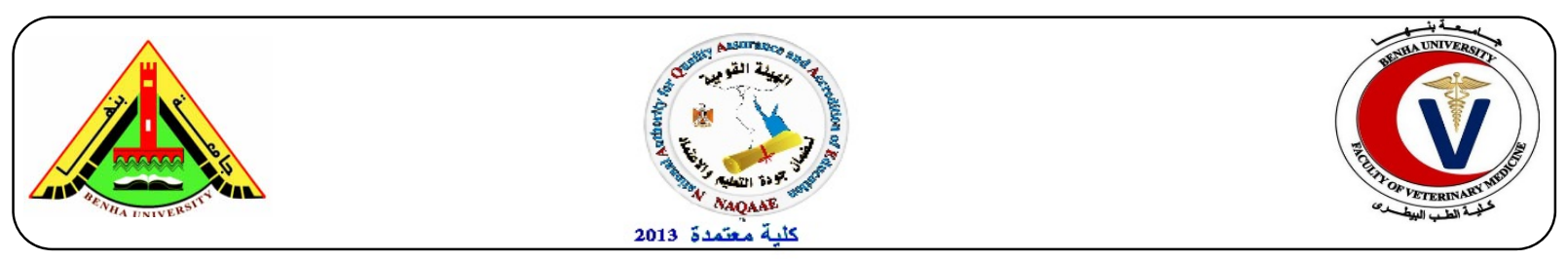

\title{
Effect of Probiotics, Prebiotics, Synbiotics, Organic Acids and Enzymes Supplementation on broiler Chicks' Immunity in relation to the Economic Performance.
}

\author{
Liza Sabry Mohammed ${ }^{1}$, Mohammed Abo-Salem, M.E. ${ }^{2}$, Atallah, S.T. ${ }^{3}$, El Shawarby, R.M. ${ }^{4}$ and Eman \\ Ramadan Kamel ${ }^{1}$
}

${ }^{1}$ Department of Animal Wealth Development, Faculty of Veterinary Medicine, Benha University. ${ }^{2}$ Department of Forensic Medicine and Toxicology, Dean of Faculty of Veterinary Medicine, Benha University. ${ }^{3}$ Department of Forensic Medicine and Toxicology, Faculty of Veterinary Medicine, Benha University. ${ }^{4}$ Department of Animal Husbandry and Animal Wealth Development, Faculty of Veterinary Medicine, Alexandria University. E-mail: dr.liza_sabry@yahoo.com

\begin{abstract}
A B S T R A C T
This study was conducted to evaluate the effect of different feed additives (Probiotics, Prebiotics, Synbiotics, Organic acids and Enzymes) on chicks' immunity (Antibody titer against Newcastle vaccine, differential leukocyte count, total proteins, albumin and globulin value) and economic efficiency analysis by using production functions under summer condition. Our results showed that, enzyme treated group recorded the highest value of white blood cells. Organic acids treated group for Ross breed showed the highest value for lymphocyte percentage. Heterophils percentage value was the highest for probiotics and synbiotics treated group. Concerning antibody titer to vaccination against Newcastle, we found that all the experimental groups had a positive effect on antibody titer. Regarding, albumin value, it was the highest for probiotic treated group, while globulin value for Cobb breed showed higher value for all treated groups in comparison to control group except for probiotic treated group. Regarding the effect of these additives on body weight and total return, we found positive relationship between feed additives and body weight and total return. On the basis of our results, it would be concluded that probiotics, prebiotic, synbiotic, organic acids and enzymes had positive effect on immunity and economic performance of broiler chicken.
\end{abstract}

Keywords: Organic acids, Enzymes, Probiotics, Prebiotics, Immunity, Economic performance.

(http://www.bvmj.bu.edu.eg)

(BVMJ-30(2): 34-44, 2016)

\section{INTRODUCTION}

$\mathrm{W}$ ith increasing ban on antibiotic usage as growth promoters feed additives in animal production due to development of antimicrobial resistance of bacteria. (Amaechi and Amaeze, 2012). So the researchers are attempting to find alternatives to antibiotics to increase production and growth performance of poultry (Khan et al., 2014). Probiotics, prebiotics, symbiotic and organic acids are considered one from widely used antibiotics (Abdel Fattah e al., 2008 and Bozkurt et al., 2009). Supplementation of probiotic to broiler diet had positive effect on immune response to Newcastle and SRBC. Moreover, $1 \mathrm{~g} / \mathrm{kg}$ supplementation of probiotic is more suitable than $1.5 \mathrm{~g} / \mathrm{kg}$ diet (Saffari Samani et al., 2012 and Nikpiran et al., 2013). Prebiotic supplemented diets had greater IgA content in the duodenum and by increasing concentration of dietary prebiotic, IgA content increased linearly
(Gao et al., 2008). Chicks fed acidified diets had better immune response as indicated by their higher serum globulin (Abdel fattah et al., 2008). The productive efficiency can be achieved when production is maximized at the lowest cost possible and when the average cost is at the lowest point on the average cost curve or using the least amount of resources to produce a given output level (Atallah, 1997). The production functions used to determine the major important variables that affect broiler production which were (starter, finisher, feed conversion, total feed, drugs, vaccines, disinfectants, veterinary supervision and total veterinary management). Therefore, the objective of this study is to evaluate the effect of different feed additives (Probiotics, Prebiotic, Symbiotic, Organic acids and Enzymes) on immunity and the economic analysis of production functions and 
their effect on the economic performance of broiler chicken of both Cobb and Ross breeds.

\section{MATERIALS AND METHODS}

\subsection{Experimental Chicks:}

Our study was carried out at Poultry Research Farm belonging to the Department of Animal Wealth Development, Faculty of Veterinary Medicine, Benha University, Egypt, in the period from $16^{\text {th }}$ June till $28^{\text {th }}$ July 2014 . A total of 576 healthy unsexed one-day-old broiler chicks (Cobb and Ross breed) were used. The Ross breed was purchased from El-Wadi Company and the Cobb breed was purchased from El-Watania Company.

\subsection{Management and Housing:}

The broiler chicks were weighed individually and wing banded, then allocated randomly in to 12 groups. Each group consisted of three replicates (total 36 replicates for all groups). They were housed in a clean, well ventilated room previously fumigated with formalin and potassium permanganate. The room was provided with heaters to adjust the environmental temperature according to the age of chicks. The floor was bedded with fresh wood shaving forming a litter with $5 \mathrm{~cm}$ of depth. Each compartment was provided by suitable feeders and waterers. Brooding temperature started at $35^{\circ} \mathrm{C}$ during the first 3 days, then $32{ }^{\circ} \mathrm{C}$ to the end of the $1^{\text {st }}$ week; $30{ }^{\circ} \mathrm{C}$ for the $2^{\text {nd }}$ week; $28^{\circ} \mathrm{C}$ throughout the $3^{\text {rd }}$ week till the end of experiment (Marwa, 2013). Natural and artificial lighting was provided for 24 hours over the experimental period. Ventilation of the rooms depended on windows and negative pressure fans.

\subsection{Vaccination:}

The chicks were vaccinated against most common viral diseases which may infect broiler chicks as shown in the table below:

\begin{tabular}{lllll}
\hline Age & Type of vaccine & Type & Route of vaccination & Company \\
\hline $7^{\text {th }}$ day & Hitchiner B1+IB & Live & Eye drop & Korea \\
$10^{\text {th }}$ day & Lasota & Killed & S/C & Ceva \\
$14^{\text {th }}$ day & Gumboro & Live & Eye drop & Intervet \\
$30^{\text {th }}$ day & Colon+IB. & Live & Drinking water & Intervet \\
\hline
\end{tabular}

\subsection{Experimental Diets:}

Chicks were fed on well-balanced diets (NRC, 1994) as described in table 3. Starter diet was given till the $14^{\text {th }}$ day of age. After that, chicks were fed on grower diet which was given till the $28^{\text {th }}$ day of age. After that, chicks were fed on finisher diet till the end of the experiment ( $42^{\text {nd }}$ day of age). Chicks were allocated as the following:

1. Group 1 received the basal diet.

2. Group 2 received the basal diet supplemented with Probiotic $(0.1 \mathrm{~g}$ Baymix ${ }^{\circledR}$ Grobig $^{\mathrm{TM}} / \mathrm{kg}$ ration).

3. Goup 3 received the basal diet supplemented with Prebiotics $(0.5 \mathrm{~g}$ CelMax dry ${ }^{\mathrm{TM}} / \mathrm{kg}$ ration).

4. Group 4 received the basal diet supplemented with Synbiotics (0.1g Baymix $+0.5 \mathrm{gm}$ Cel-max dry $/ \mathrm{kg}$ ration).

5. Group 5 received the basal diet supplemented with Organic acids (1g Fylax ${ }^{\circledR}$ plus / kg ration).

6. Group 6 received a diet supplemented with enzymes $\left(0.2 \mathrm{~g}\right.$ Allzyme ${ }^{\circledR} \mathrm{SSF} / \mathrm{kg}$ ration).
The Ingredients and the chemical composition of the diets are represented in tables 1,2 and 3 .

\subsection{Evaluation of immune response:}

\subsubsection{Blood Sampling}

About $1 \sim 2 \mathrm{ml}$ of blood from the birds were aseptically collected from the jugular vein/wing vein with a sterile $2 \mathrm{ml}$ disposable syringe. Blood samples were collected at zero day, $1^{\text {st }}, 2^{\text {nd }}$ and $3^{\text {rd }}$ week post vaccination and at the end of growing period. About $0.5-1 \mathrm{ml}$ of blood was taken in a vial containing EDTA as anticoagulant at $1 \mathrm{mg} / \mathrm{ml}$, for estimation of hematological parameters.

\subsubsection{Hematological parameters measurement:}

Hematological variables including white blood cells (WBCs) and red blood cells (RBCs) were performed in a Neubauer hemocytometer using a 1:200 dilutions with Natt and Herrick solution. Differential leukocyte count, hemoglobin ( $\mathrm{Hgb})$ concentration, packed cell volume (PCV) were determined as described previously (Campbell, 1995).

\subsubsection{Haemagglutination inhibition (HI) test:}




\subsubsection{Serum samples and preparation:}

Blood samples were collected at the fifth day of age from starting of the experiment, and then taken weekly for 3 successive weeks. Clotted blood samples were centrifuged at $3000 \mathrm{rpm}$. for 15 minutes to obtain clear serum. The serum samples were kept in small labelled sterile tubes and stored at $-20^{\circ} \mathrm{C}$ till used (Stoot and Fellah, 1983).

\subsubsection{Reagents:}

Reagents used in the HI test were prepared according to the standard microplate system described by Majiyagble and Hitchner (1977) as follow: Phosphate buffer saline $\mathrm{pH}$.

Virus antigen: Newcastle disease virus (NDV). Live Hitchner vaccine. The virus was previously titrated and adjusted to 4 HAU/50 $\mu \mathrm{l}$ (Haemagglutination unit). Chicken RBCs suspension (1\% in PBS pH).

Blood was collected from the wing vein of a chick in a centrifuge tube containing EDTA as anticoagulant. The red cells were washed by centrifugation three times with sterile physiological saline. The RBCs suspension (1\%) was prepared by adding $1 \mathrm{ml}$ of washed RBCs to $99 \mathrm{ml}$ PBS-pH to be used in the HI test.

\subsubsection{Equipment:}

96 well microtiter plates of U-shaped bottom (Greiner bio-one ${ }^{\circledR}$, Germany).

Multichannel microtiter pipette of 10-200 $\mu \mathrm{l}$ capacity (Costar ${ }^{\circledR}$, USA).

\subsubsection{Method of HI test:}

$\mathrm{HI}$ test was performed as the following: Using the multichannel microtiter pipette, $50 \mu 1$ of PBS-pH were dispensed in each well of the 96-well microtiter plates. $50 \mu \mathrm{l}$ of each serum sample (all serum samples of all group) from the beginning till the end of the experiment were dispensed in the first well of plates (one column in each plate was left as RBCs control).

Two-fold serial dilutions of the serum samples were applied along the column length to generate eight consequent dilutions. $50 \mu \mathrm{l}$ of the pre-diluted virus antigen were added to all wells of the plates except the control column. Plates were incubated at room temperature for 60 minutes. 50 $\mu 1$ of chicken RBCs suspension (1\%) were added to all wells of the plates (including the control). Plates were incubated at room temperature for 1530 minutes before recording the results.

The HI titres were expressed as the reciprocal of the highest dilution showing complete hemagglutination inhibition activity (appearance of button shape).

\subsection{Biochemical analysis of blood:}

Total protein of serum was determined by using chem 7 and albumin also was determined.

\subsection{Economical analysis:}

Production function: The production functions were used to assess the effect of changes in production and costs parameters on broiler production and return by using forward, backward, enter and mixer methods by using SPSS/PC+ (2004). Was carried out in the forms of linear and logarithmic forms according to (Doll and Orazem ,1978; Afifi ,1988 and Atallah ,1997). Aimed to estimate the effect of feed additives on body weight of broiler for each group and all groups by the two forms of the function (linear and logarithmic). Choosing the best function of either production or costs was done according to the acceptance of the function economically, statistically (significance of $F$ test, $t$ - test as well as value of adjusted coefficient of determination $\mathrm{R}^{2}$ ) and reality of its results to broiler production (Atallah, 1994 and 1997).

\subsection{Statistical Analysis:}

Differences between studied groups and breeds were analyzed by using One-Way ANOVA and Duncan's multiple comparison Post Hoc tests (Duncan, 1955). Statistical analysis was performed using the statistical software package SPSS for Windows SPSS/PC PC $^{+}$version 16"(SPSS, 2004). Statistical significance between mean values was set at $(P<0.05)$. Data were reported as means and standard error.

\section{RESULTS}

\subsection{Effect of different treatments among different breeds on Hematological parameters of broiler chickens:}

Result in table (4) clarifies that hemoglobin value, erythrocytes, packed cell volume and white blood cells values differed significantly $(p<0.05)$ among different breeds.

\subsection{Effect of different treatments among different breeds on differential Leukocyte Count of broiler chickens:}

Regarding the values of Heterophils \%, Lymphocyte $\%$, monocyte $\%$, basophile $\%$ and esinophile \% (table, 5) there were a significant 
difference at $(p<0.05)$ for both Cobb and Ross breeds.

\subsection{Effect of different feed additives on antibody titer against Newcastle disease virus:}

Result in table (6) showed that antibody titer was significantly differed $(p<0.05)$ among different groups and breeds.

\subsection{Effect of different feed additives on biochemical parameters of blood}

Result in table (7) clarifies non-significant differences $(p>0.05)$ among different breeds on globulin, total protein and albumin to globulin values.

\subsection{Effect of different feed additives on Production functions:}

Results in Table (8) the results revealed that the logarithmic production function was significant $(P<0.05)$, and about $70 \%$ from the changes in body weight were attributed to changes in production resources.

Table 1: Ingredients of starter, grower and finisher diets (Basal diet).

\begin{tabular}{|c|c|c|c|}
\hline Ingredients (\%) & Starter & Grower & Finisher \\
\hline Corn grain & 53.55 & 52.88 & 59.46 \\
\hline $\begin{array}{l}\text { Soyabean }(44 \%) \\
\text { protein }\end{array}$ & 33.2 & 31.10 & 25.5 \\
\hline Corn gluten meal & 5.5 & 5.60 & 5.5 \\
\hline Vegetable oil & 2.85 & 5.85 & 5.40 \\
\hline $\begin{array}{l}\text { Di-calcium } \\
\text { phosphate }\end{array}$ & 2.03 & 1.85 & 1.825 \\
\hline Limestone & 1.18 & 1.17 & 0.95 \\
\hline L-Lysine & 0.50 & 0.4550 & 0.335 \\
\hline D-L methionine & 0.33 & 0.24 & 0.20 \\
\hline Sodium chloride & 0.30 & 0.30 & 0.30 \\
\hline Vit \&min premix ${ }^{(1)}$ & 0.30 & 0.30 & 0.30 \\
\hline $\begin{array}{l}\text { Sodium } \\
\text { bicarbonate }\end{array}$ & 0.15 & 0.15 & 0.15 \\
\hline L- threonine & 0.12 & 0.10 & 0.08 \\
\hline
\end{tabular}

${ }^{(1)}$ Purchased by AGRI-VIT $10^{\text {th }}$ of Ramadan city, Egypt . Each $3 \mathrm{~kg}$ contains contain: Vitamin $\mathrm{A}=$ $12,000,000 \mathrm{IU}, \mathrm{D}_{3}=2,000,000 \mathrm{IU}, \mathrm{E}=10,000 \mathrm{mg}$, $\mathrm{K}_{3}=2000 \mathrm{mg}, \mathrm{B}_{1}=1000 \mathrm{mg}, \mathrm{B}_{2}=5000 \mathrm{mg}, \mathrm{B}_{6}=1500$ $\mathrm{mg}, \mathrm{B}_{12}=10 \mathrm{mg}$, Biotin $=50 \mathrm{mg}$, pantothenic acid= $10000 \mathrm{mg}$, Nicotinic acid $=30000 \mathrm{mg}$, Folic acid $=1000 \mathrm{mg}$, Zinc $=50,000 \mathrm{mg}$, Manganese $=60,000$ $\mathrm{mg}$, Iron $=30,000 \mathrm{mg}$, Copper $=10,000 \mathrm{mg}$, Iodine $=1,000 \mathrm{mg}$, Selenium $=100 \mathrm{mg}$, Cobalt $=100 \mathrm{mg}$, Cobalt $=1000 \mathrm{mg}$, and Calcium carbonate up to $3 \mathrm{Kg}$.
Table 2: Ingredients of starter, grower and finisher (Energy Enzyme diet).

\begin{tabular}{|c|c|c|c|}
\hline Ingredients (\%) & Starter & Grower & Finisher \\
\hline Corn grain & 55.36 & 54.85 & 61.33 \\
\hline $\begin{array}{l}\text { Soyabean }(44 \%) \\
\text { protein }\end{array}$ & 32.90 & 30.80 & 25.20 \\
\hline Corn gluten meal & 5.50 & 5.50 & 5.5 \\
\hline Vegetable oil & 1.30 & 4.25 & 3.80 \\
\hline $\begin{array}{l}\text { Di-calcium } \\
\text { phosphate }\end{array}$ & 2.03 & 1.825 & 1.825 \\
\hline Limestone & 1.20 & 1.20 & 0.95 \\
\hline L-Lysine & 0.50 & 0.46 & 0.34 \\
\hline D-L methionine & 0.33 & 0.24 & 0.20 \\
\hline Sodium chloride & 0.30 & 0.30 & 0.30 \\
\hline Vit \&min premix ${ }^{(1)}$ & 0.30 & 0.30 & 0.30 \\
\hline $\begin{array}{l}\text { Sodium } \\
\text { bicarbonate }\end{array}$ & 0.15 & 0.15 & 0.15 \\
\hline L- threonine & 0.12 & 0.10 & 0.08 \\
\hline Enzyme & 0.20 & 0.20 & 0.20 \\
\hline
\end{tabular}

(1) Purchased by AGRI-VIT $10^{\text {th }}$ of Ramadan city, Egypt. Each $3 \mathrm{~kg}$ contains contain: Vitamin $\mathrm{A}=$ $12,000,000 \mathrm{IU}, \mathrm{D}_{3}=2,000,000 \mathrm{IU}, \mathrm{E}=10,000$ $\mathrm{mg}, \mathrm{K}_{3}=2000 \mathrm{mg}, \mathrm{B}_{1}=1000 \mathrm{mg}, \mathrm{B}_{2}=5000 \mathrm{mg}$, $\mathrm{B}_{6}=1500 \mathrm{mg}, \mathrm{B}_{12}=10 \mathrm{mg}$, Biotin $=50 \mathrm{mg}$, pantothenic acid $=10000 \mathrm{mg}$, Nicotinic acid $=$ $30000 \mathrm{mg}$, Folic acid $=1000 \mathrm{mg}$, Zinc $=50,000$ $\mathrm{mg}$, Manganese $=60,000 \mathrm{mg}$, Iron $=30,000 \mathrm{mg}$, Copper $=10,000 \mathrm{mg}$, Iodine $=1,000 \mathrm{mg}$, Selenium $=100 \mathrm{mg}$, Cobalt $=100 \mathrm{mg}$, Cobalt $=$ $1000 \mathrm{mg}$, and Calcium carbonate up to $3 \mathrm{Kg}$.

Table 3: Chemical composition of starter, grower and finisher diets.

\begin{tabular}{llll}
\hline Item & Starter & Grower & Finisher \\
\hline $\begin{array}{l}\text { Crude } \\
\text { protein\% }\end{array}$ & 22 & 21 & 19 \\
M En (kcal/kg) & 3000 & 3177 & 3225 \\
$\begin{array}{l}\text { Lysine\% } \\
\text { Methionine+ }\end{array}$ & 1.35 & 1.27 & 1.05 \\
$\begin{array}{l}\text { cysteine\% } \\
\text { Calcium \% }\end{array}$ & 1.05 & 0.94 & 0.85 \\
$\begin{array}{l}\text { Available } \\
\text { phosphorus \% }\end{array}$ & 0.50 & 0.46 & 0.45 \\
$\begin{array}{l}\text { Chloride \% } \\
\text { Na \% }\end{array}$ & 0.22 & 0.22 & 0.22 \\
\hline
\end{tabular}

Calculated according to NRC, 1994. 
Table (4): Effect of different treatments among different breeds on Hematological parameters of broiler chickens (Mean \pm SE).

\begin{tabular}{|c|c|c|c|c|c|c|}
\hline Breed & Group & $\mathrm{N}$ & $\begin{array}{l}\mathrm{Hb} \\
(\mathrm{gm} / \mathrm{dL})\end{array}$ & $\begin{array}{l}\text { RBCs } \\
\left(10^{6} / \mu L\right)\end{array}$ & PCV\% & $\begin{array}{l}\text { WBCs } \\
\left(10^{3} / \mu \mathrm{L}\right)\end{array}$ \\
\hline \multirow{11}{*}{$\frac{\hat{0}}{0}$} & \multirow[t]{2}{*}{ Control } & \multirow[t]{2}{*}{5} & $5.89^{d}$ & $1.70^{\mathrm{d}}$ & $25.20^{\mathrm{d}}$ & $30.10^{\mathrm{b}}$ \\
\hline & & & \pm 0.46 & \pm 0.14 & \pm 0.86 & \pm 3.52 \\
\hline & \multirow[t]{2}{*}{ Probiotics } & \multirow[t]{2}{*}{5} & $5.59^{d}$ & $1.69^{d}$ & $25.20^{\mathrm{d}}$ & $36.0^{\mathrm{ab}}$ \\
\hline & & & \pm 0.46 & \pm 0.06 & \pm 0.80 & \pm 5.37 \\
\hline & \multirow[t]{2}{*}{ Prebiotics } & \multirow[t]{2}{*}{5} & $9.48^{\mathrm{b}}$ & $1.68^{\mathrm{d}}$ & $25.50^{\mathrm{d}}$ & $13.75^{\mathrm{c}}$ \\
\hline & & & \pm 0.98 & \pm 0.11 & \pm 1.04 & \pm 2.43 \\
\hline & \multirow[t]{2}{*}{ Synbiotics } & \multirow[t]{2}{*}{5} & $9.07^{\mathrm{b}}$ & $1.93^{\mathrm{c}}$ & $27.67^{\mathrm{c}}$ & $15.07^{\mathrm{c}}$ \\
\hline & & & \pm 0.97 & \pm 0.20 & \pm 1.20 & \pm 0.47 \\
\hline & \multirow[t]{2}{*}{ Organic acids } & \multirow[t]{2}{*}{5} & $10.77^{\mathrm{ab}}$ & $2.12^{\mathrm{c}}$ & $29.67^{\mathrm{b}}$ & $16.43^{\mathrm{c}}$ \\
\hline & & & \pm 0.63 & \pm 0.04 & \pm 0.67 & \pm 0.84 \\
\hline & \multirow[t]{4}{*}{ Enzymes } & \multirow[t]{2}{*}{5} & $11.60^{\mathrm{a}}$ & $6.55^{\mathrm{a}}$ & $39.60^{\mathrm{a}}$ & $37.64^{\mathrm{ab}}$ \\
\hline \multirow{3}{*}{ Total } & & & \pm 1.03 & \pm 0.29 & \pm 2.06 & \pm 2.02 \\
\hline & & \multirow[t]{2}{*}{30} & $8.51^{\mathrm{B}}$ & $2.74^{\mathrm{A}}$ & $28.96^{\mathrm{A}}$ & $26.73^{\mathrm{A}}$ \\
\hline & & & \pm 0.57 & \pm 0.40 & \pm 1.22 & \pm 2.40 \\
\hline \multirow{11}{*}{ 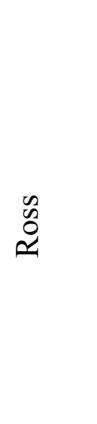 } & \multirow[t]{2}{*}{ Control } & \multirow[t]{2}{*}{5} & $7.55^{\mathrm{c}}$ & $2.35^{\mathrm{c}}$ & $27.40^{\mathrm{c}}$ & $14.50^{\mathrm{c}}$ \\
\hline & & & \pm 0.21 & \pm 0.22 & \pm 1.03 & \pm 1.80 \\
\hline & \multirow[t]{2}{*}{ Probiotics } & \multirow[t]{2}{*}{5} & $6.60^{\mathrm{d}}$ & $1.93^{\mathrm{c}}$ & $27.40^{\mathrm{c}}$ & $13.80^{\mathrm{c}}$ \\
\hline & & & \pm 0.37 & \pm 0.12 & \pm 0.75 & \pm 1.26 \\
\hline & \multirow[t]{2}{*}{ Prebiotics } & \multirow[t]{2}{*}{5} & $9.78^{\mathrm{ab}}$ & $1.96^{\mathrm{c}}$ & $27.0^{\mathrm{c}}$ & $14.75^{\mathrm{c}}$ \\
\hline & & & \pm 0.82 & \pm 0.05 & \pm 0.00 & \pm 1.11 \\
\hline & \multirow[t]{2}{*}{ Synbiotics } & \multirow[t]{2}{*}{5} & $9.0^{\mathrm{b}}$ & $1.82^{\mathrm{c}}$ & $27.0^{\mathrm{c}}$ & $19.70^{c}$ \\
\hline & & & \pm 0.76 & \pm 0.16 & \pm 1.53 & \pm 2.16 \\
\hline & \multirow[t]{2}{*}{ Organic acids } & \multirow[t]{2}{*}{5} & $11.10^{\mathrm{ab}}$ & $2.12^{\mathrm{c}}$ & $29.33^{\mathrm{b}}$ & $17.67^{\mathrm{c}}$ \\
\hline & & & \pm 0.10 & \pm 0.06 & \pm 0.33 & \pm 0.88 \\
\hline & \multirow[t]{4}{*}{ Enzymes } & \multirow[t]{2}{*}{5} & $9.20^{\mathrm{b}}$ & $5.26^{\mathrm{b}}$ & $32.0^{\mathrm{b}}$ & $39.60^{\mathrm{a}}$ \\
\hline \multirow{3}{*}{ Total } & & & \pm 0.20 & \pm 0.19 & \pm 0.63 & \pm 2.98 \\
\hline & & \multirow[t]{2}{*}{30} & $8.65^{\mathrm{A}}$ & $2.69^{\mathrm{A}}$ & $28.44^{\mathrm{B}}$ & $20.42^{\mathrm{B}}$ \\
\hline & & & \pm 0.34 & \pm 0.27 & \pm 0.49 & \pm 2.12 \\
\hline
\end{tabular}

Means within the same column carrying different superscripts are significant at $(P \leq 0.05)$.

Table (5): Effect of different treatments among different breeds on differential Leukocyte Count of broiler chickens (Mean \pm SE).

\begin{tabular}{|c|c|c|c|c|c|c|c|}
\hline BREED & GROUP & $\mathrm{N}$ & $\begin{array}{c}\text { Heterophile } \\
\%\end{array}$ & $\begin{array}{c}\text { Lymphocyte } \\
\%\end{array}$ & $\begin{array}{c}\text { Monocyte } \\
\%\end{array}$ & $\begin{array}{c}\text { Basophile } \\
\%\end{array}$ & $\begin{array}{c}\text { Esinophile } \\
\%\end{array}$ \\
\hline \multirow{5}{*}{$\frac{0}{0}$} & Control & 5 & $24.60^{\mathrm{b}} \pm 0.81$ & $62.60^{\mathrm{ab}} \pm 2.32$ & $7.40^{\mathrm{a}} \pm 0.93$ & $0.2^{\mathrm{a}} \pm 0.20$ & $5.20^{\mathrm{b}} \pm 1.88$ \\
\hline & Probiotics & 5 & $30.20^{\mathrm{ab}} \pm 0.86$ & $52.80^{c} \pm 3.25$ & $7.20^{\mathrm{a}} \pm 1.20$ & 0 & $9.80^{\mathrm{a}} \pm 2.63$ \\
\hline & Prebiotics & 5 & $30.50^{\mathrm{ab}} \pm 2.40$ & $64.00^{\mathrm{ab}} \pm 3.24$ & $3.75^{\mathrm{c}} \pm 1.11$ & 0 & $1.75^{\mathrm{b}} \pm 0.48$ \\
\hline & Synbiotics & 5 & $31.33^{\mathrm{ab}} \pm 5.24$ & $62.00^{\mathrm{ab}} \pm 6.11$ & $4.00^{b} \pm 1.15$ & 0 & $2.67^{\mathrm{b}} \pm 1.67$ \\
\hline & $\begin{array}{l}\text { Organic } \\
\text { acids }\end{array}$ & 5 & $31.00^{\mathrm{ab}} \pm 1.00$ & $63.33^{\mathrm{ab}} \pm 2.03$ & $4.33^{\mathrm{b}} \pm 1.45$ & 0 & $2.00^{\mathrm{b}} \pm 0.58$ \\
\hline \multirow{3}{*}{ Total } & Enzymes & 5 & $28.20^{\mathrm{ab}} \pm 1.28$ & $63.40^{\mathrm{ab}} \pm 1.08$ & $5.40^{\mathrm{ab}} \pm 0.24$ & $0.4^{\mathrm{a}} \pm 0.24$ & $2.60^{\mathrm{b}} \pm 0.40$ \\
\hline & & 30 & $28.96^{\mathrm{A}} \pm 0.86$ & $61.04^{\mathrm{A}} \pm 1.38$ & $5.60^{\mathrm{A}} \pm 0.48$ & $0.12^{\mathrm{A}} \pm 0.07$ & $4.36^{\mathrm{A}} \pm 0.87$ \\
\hline & Control & 5 & $25.60^{\mathrm{b}} \pm 1.96$ & $65.00^{\mathrm{ab}} \pm 2.26$ & $6.80^{\mathrm{ab}} \pm 0.58$ & 0 & $2.60^{\mathrm{b}} \pm 0.40$ \\
\hline \multirow{5}{*}{$\begin{array}{l}n \\
2 \\
2 \\
2\end{array}$} & Probiotics & 5 & $34.60^{\mathrm{a}} \pm 2.48$ & $57.80^{\mathrm{b}} \pm 2.60$ & $5.00^{\mathrm{ab}} \pm 0.32$ & 0 & $2.60^{\mathrm{b}} \pm 0.40$ \\
\hline & Prebiotics & 5 & $30.75^{\mathrm{ab}} \pm 1.44$ & $62.75^{\mathrm{ab}} \pm 1.18$ & $4.00^{b} \pm 0.91$ & 0 & $2.50^{\mathrm{b}} \pm 0.65$ \\
\hline & Synbiotics & 5 & $34.00^{\mathrm{a}} \pm 4.36$ & $60.00^{\mathrm{ab}} \pm 5.77$ & $3.67^{c} \pm 0.88$ & 0 & $2.33^{b} \pm 0.67$ \\
\hline & $\begin{array}{l}\text { Organic } \\
\text { acids }\end{array}$ & 5 & $28.00^{\mathrm{ab}} \pm 2.08$ & $69.33^{a} \pm 1.76$ & $1.67^{\mathrm{d}} \pm 0.33$ & 0 & $1.00^{\mathrm{b}} \pm 0.00$ \\
\hline & Enzymes & 5 & $28.40^{\mathrm{ab}} \pm 1.12$ & $63.20^{\mathrm{ab}} \pm 1.39$ & $5.40^{\mathrm{ab}} \pm 0.51$ & $0.40^{\mathrm{a}} \pm 0.24$ & $2.60^{\mathrm{b}} \pm 0.40$ \\
\hline Total & & 30 & $30.08^{\mathrm{A}} \pm 1.06$ & $62.76^{\mathrm{A}} \pm 1.16$ & $4.72^{\mathrm{A}} \pm 0.38$ & $0.08^{\mathrm{A}} \pm 0.06$ & $2.36^{\mathrm{B}} \pm 0.20$ \\
\hline
\end{tabular}


Effect of Probiotics, Prebiotics, Synbiotics, Organic Acids and Enzymes on broiler Chicks' Immunity

Table (6): Effect of different feed additives on antibody titer against Newcastle disease virus (Mean $\pm \mathrm{SE}$ ).

\begin{tabular}{llllll}
\hline Breed & Group & Zero & $1^{\text {st }}$ week & $2^{\text {nd }}$ week & $3^{\text {rd }}$ week \\
\hline Cobb & Control & $2.82^{\mathrm{a}} \pm 0.11$ & $1.88^{\mathrm{b}} \pm 0.11$ & $1.14^{\mathrm{cd}} \pm 0.10$ & $2.14^{\mathrm{bc}} \pm 0.10$ \\
& Probiotics & $2.60^{\mathrm{ab}} \pm 0.11$ & $2.08^{\mathrm{ab}} \pm 0.10$ & $1.48^{\mathrm{ab}} \pm 0.10$ & $2.47^{\mathrm{a}} \pm 0.10$ \\
& Prebiotics & $2.89^{\mathrm{a}} \pm 0.10$ & $1.90^{\mathrm{ab}} \pm 0.10$ & $1.33^{\mathrm{bc}} \pm 0.10$ & $2.26^{\mathrm{abc}_{ \pm}} \pm 0.13$ \\
& Synbiotics & $2.79^{\mathrm{a}} \pm 0.11$ & $2.05^{\mathrm{ab}} \pm 0.10$ & $1.26^{\mathrm{bc}} \pm 0.10$ & $2.17^{\mathrm{abc}_{ \pm}} \pm 0.10$ \\
& Organic acids & $2.31^{\mathrm{b}} \pm 0.13$ & $1.99^{\mathrm{ab}} \pm 0.10$ & $1.36^{\mathrm{bc}} \pm 0.10$ & $2.17^{\mathrm{abc}_{ \pm}} \pm 0.10$ \\
& Enzymes & $2.41^{\mathrm{b}} \pm 0.13$ & $1.99^{\mathrm{ab}} \pm 0.10$ & $1.29^{\mathrm{bc}} \pm 0.10$ & $2.07^{\mathrm{c}} \pm 0.11$ \\
Ross & Control & $2.56^{\mathrm{ab}} \pm 0.11$ & $1.87^{\mathrm{b}} \pm 0.10$ & $1.48^{\mathrm{abc}} \pm 0.10$ & $2.14^{\mathrm{b}} \pm 0.10$ \\
& Probiotics & $2.62^{\mathrm{ab}} \pm 0.10$ & $2.11^{\mathrm{ab}} \pm 0.10$ & $1.63^{\mathrm{ab}} \pm 0.10$ & $2.41^{\mathrm{b}} \pm 0.11$ \\
& Prebiotics & $2.56^{\mathrm{ab}} \pm 0.11$ & $1.93^{\mathrm{ab}} \pm 0.10$ & $1.33^{\mathrm{bc}} \pm 0.10$ & $2.22^{\mathrm{abc}} \pm 0.11$ \\
& Synbiotics & $2.82^{\mathrm{a}} \pm 0.09$ & $2.11^{\mathrm{ab}} \pm 0.10$ & $1.78^{\mathrm{a}} \pm 0.10$ & $2.23^{\mathrm{abc}} \pm 0.10$ \\
& Organic acids & $2.80^{\mathrm{a}} \pm 0.10$ & $1.93^{\mathrm{ab}} \pm 0.10$ & $1.60^{\mathrm{ab}} \pm 0.10$ & $2.41^{\mathrm{ab}} \pm 0.10$ \\
& Enzymes & $2.61^{\mathrm{ab}} \pm 0.13$ & $2.22^{\mathrm{a}} \pm 0.11$ & $0.90^{\mathrm{d}} \pm 0.10$ & $2.35^{\mathrm{abc}_{ \pm}} \pm 0.10$ \\
\hline
\end{tabular}

Means within the same column carrying different superscripts are significant at $(P \leq 0.05)$.

Table (7): Effect of different treatments among different breeds on biochemical parameters of blood of broiler chickens (Mean $\pm \mathrm{SE})$.

\begin{tabular}{cllllll}
\hline BREED & GROUP & $\mathrm{N}$ & $\begin{array}{l}\text { Albumin } \\
(\mathrm{g} / \mathrm{dL})\end{array}$ & $\begin{array}{l}\text { Globulin } \\
(\mathrm{g} / \mathrm{dL})\end{array}$ & $\begin{array}{l}\text { Total protein } \\
(\mathrm{g} / \mathrm{dL})\end{array}$ & $\mathrm{A} / \mathrm{G}$ ratio \\
\hline & Control & 5 & $1.69^{\mathrm{b}} \pm 0.07$ & $1.39^{\mathrm{a}} \pm 0.14$ & $3.08^{\mathrm{a}} \pm 0.18$ & $1.27^{\mathrm{b}} \pm 0.13$ \\
& Probiotics & 5 & $2.30^{\mathrm{a}} \pm 0.37$ & $1.23^{\mathrm{a}} \pm 0.11$ & $3.53^{\mathrm{a}} \pm 0.29$ & $2.01^{\mathrm{a}} \pm 0.54$ \\
$\overline{0}$ & Prebiotics & 5 & $1.54^{\mathrm{b}} \pm 0.13$ & $1.72^{\mathrm{a}} \pm 0.25$ & $3.26^{\mathrm{a}} \pm 0.34$ & $0.94^{\mathrm{b}} \pm 0.09$ \\
0 & Synbiotics & 5 & $1.68^{\mathrm{b}} \pm 0.15$ & $1.57^{\mathrm{a}} \pm 0.20$ & $3.25^{\mathrm{a}} \pm 0.27$ & $1.14^{\mathrm{b}} \pm 0.19$ \\
& Organic acids & 5 & $1.48^{\mathrm{b}} \pm 0.05$ & $1.72^{\mathrm{a}} \pm 0.42$ & $3.20^{\mathrm{a}} \pm 0.37$ & $1.02^{\mathrm{b}} \pm 0.22$ \\
& Enzymes & 5 & $1.79^{\mathrm{b}} \pm 0.13$ & $1.85^{\mathrm{a}} \pm 0.26$ & $3.64^{\mathrm{a}} \pm 0.30$ & $1.02^{\mathrm{b}} \pm 0.16$ \\
Total & & 30 & $1.73^{\mathrm{a}} \pm 0.08$ & $1.58^{\mathrm{A}} \pm 0.10$ & $3.31^{\mathrm{A}} \pm 0.11$ & $1.22^{\mathrm{A}} \pm 0.11$ \\
& Control & 5 & $1.64^{\mathrm{b}} \pm 0.06$ & $1.90^{\mathrm{a}} \pm 0.24$ & $3.54^{\mathrm{a}} \pm 0.24$ & $0.93^{\mathrm{b}} \pm 0.14$ \\
& Probiotics & 5 & $1.83^{\mathrm{b}} \pm 0.16$ & $1.82^{\mathrm{a}} \pm 0.28$ & $3.65^{\mathrm{a}} \pm 0.26$ & $1.11^{\mathrm{b}} \pm 0.25$ \\
$\tilde{0}$ & Prebiotics & 5 & $1.73^{\mathrm{b}} \pm 0.12$ & $1.78^{\mathrm{a}} \pm 0.44$ & $3.52^{\mathrm{a}} \pm 0.36$ & $1.22^{\mathrm{b}} \pm 0.35$ \\
$\simeq$ & Synbiotics & 5 & $1.82^{\mathrm{b}} \pm 0.13$ & $1.56^{\mathrm{a}} \pm 0.19$ & $3.38^{\mathrm{a}} \pm 0.17$ & $1.23^{\mathrm{b}} \pm 0.16$ \\
& Organic acids & 5 & $1.63^{\mathrm{b}} \pm 0.08$ & $1.83^{\mathrm{a}} \pm 0.13$ & $3.46^{\mathrm{a}} \pm 0.18$ & $0.90^{\mathrm{b}} \pm 0.05$ \\
& Enzymes & 5 & $1.94^{\mathrm{ab}} \pm 0.06$ & $1.88^{\mathrm{a}} \pm 0.17$ & $3.82^{\mathrm{a}} \pm 0.16$ & $1.07^{\mathrm{b}} \pm 0.12$ \\
Total & & 30 & $1.77^{\mathrm{A}} \pm 0.04$ & $1.79^{\mathrm{A}} \pm 0.09$ & $3.56^{\mathrm{A}} \pm 0.09$ & $1.08^{\mathrm{A}} \pm 0.07$ \\
\hline
\end{tabular}

Means within the same column carrying different superscripts are significant at $(P \leq 0.05)$.

Table (8): Production function of final body weight and production resources (feed cost, additive cost and drug cost)

\begin{tabular}{|c|c|}
\hline $\begin{array}{l}\text { Function } \\
\mathrm{T} \\
\mathrm{F} \\
\mathrm{R}^{-2}\end{array}$ & 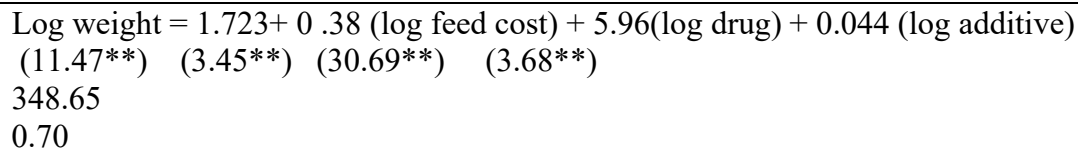 \\
\hline
\end{tabular}

*** Significant at $(P<0.05)$.

Table (9): Production function of total cost (TC) and additive cost.

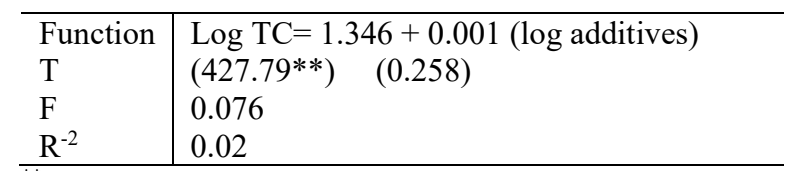

${ }^{* *}$ Significant at $(P<0.05)$. 
Table (10): Production function of total return (TR) and feed additives.

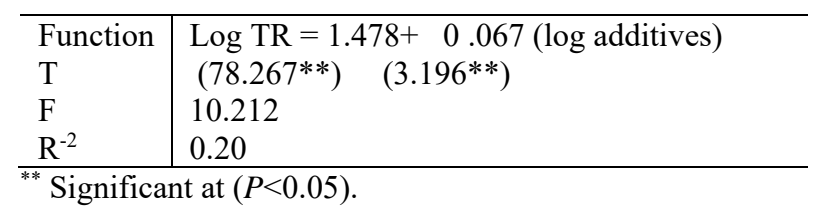

\section{DISCUSSION}

Regarding hemoglobin value, it was higher for both breed of prebiotic, symbiotic, organic acid and enzyme treated groups than the control group. These results agreed with Beski and Al sardary (2015) who found that value of HB \% was high in synbiotic treated group compared to probiotic and control group. Concerning value of RBCS, there was a significant difference $(p<0.05)$ for both Cobb and Ross breeds. The highest value was found for enzyme treated of both breeds followed by organic acid treated of both breed $\left(2.12 \times 10^{6 / \mu l}\right)$ while the lowest value was found for prebiotic group of Cobb breed $\left(1.68 \times 10^{6 / \mu 1}\right)$, this result disagreed with Khosravi et al. (2010) who found that erythrocyte was lower in organic acid than control group.

Value of packed cell volume varied significantly $(p<0.05)$ among different treated group of both breeds. The highest value was found for enzyme treated group of both breed (39.60 and 32.00 for Cobb and Ross breed respectively) while the lowest value was found for probiotic and control groups for Cobb breed (25.20). These results agreed with Beski and Al sardary (2015) who found that value of $\mathrm{PCV} \%$ was the lowest in probiotic treated group. Also, Amer (2014) found a slight increase in all hematological parameters of birds fed diets supplemented with phytase.

Value of white blood cells varied significantly $((p<0.05)$ among different treated group of both breeds. The highest value was found for enzyme treated group of both breed (39.60 and 37.64 of Ross and Cobb breed respectively) and also for probiotic treated group of Cobb breed (36.0). While the lowest value was found for prebiotic treated group of Cobb breed (13.75).

These results were in agreement with Khosravi et al. (2010) who found that leukocyte count was high for probiotic treated compared to control group and the lowest value was found in organic acid. Also, Amer (2014) found a slight increase in WBCS in diets supplemented with phytase.

Value of hemoglobin, red blood cells, packed cell volume and white blood cells differed significantly among different breeds. Hemoglobin was higher in Ross breed than Cobb breed, while white blood cells and packed cell volume were higher in Cobb breed than Ross breed, and these results in agreement with (Talebi et al, 2005) who reported the same trend.

Result in table (5) cleared that differential Leukocyte Count was differed significantly $(p<0.05)$ among different weeks and treated group. Regarding heterophils percentage value, it was higher in all experimental groups of both breed than control group. The highest value was found for probiotic group of Ross breed (34.60\%) and the lowest value was found for control group of $\mathrm{Cobb}$ breed (24.60).

Concerning value of Lymphocyte percentage, there was a significant difference $(\mathrm{p}<0.05)$ for both Cobb and Ross breeds. The highest value was found for organic acid group of Ross breed $(69.33 \%)$ and the lowest value was found for probiotic group of Cobb breed (52.80). These results were agreed with Haque et al. (2010), who stated that the lymphocyte cells of broilers were increased suggesting an increased level of immunity with organic acid supplementation. Also, Al Saad et al .2014) reported that there was significant increase in number of White Blood Cells (WBC) in blood samples of organic acids group compared to antibiotic group. These results were also in agreement with Chen et al. (2005) who indicated that organic acid could stimulate immune response and increase resistance to microbial pathogens as they were utilized in broilers diet. Acidifiers inhibit joining pathogenic bacteria to intestinal mucosa and creating acidic environment in intestine. Also, Roser (2006) proved that adding organic acids to broiler diets increase immunity response this occurred via stimulation or activation of immune cells by these feed additives.

The value of monocyte percentage varied significantly $(p<0.05)$ among different treated group. The highest value was found for control group and probiotic treated of Cobb breed (7.40 $\%$ and 7.20) and the lowest value was found for organic acid group of Ross breed (1.67). These results were in agreement with Salim et al. (2012) who found that white blood cell and monocyte levels were significantly higher in the bacillus subtilis compared with the control. Regarding 
organic acid addition, Mahdavi and Torki (2009) noted that the dietary inclusion of organic acid did not affect the counts of monocyte, at days 21, 42 and 49 of broilers life.

Concerning the value of basophile \%, the highest value found for enzyme treated of both breed $(0.40 \%)$. Concerning value of eosinophils $\%$, the highest value was found for probiotic treated group of Cobb breed (9.80\%) and the lowest value was found for organic acid treated of Ross breed $(1.0 \%)$. This result agreed with Khosravi et al (2010) who found that eosinophils \% was high for probiotic treated group, while for organic acid it was the same as control group.

Result in table (6) showed that antibody titer at $3^{\text {rd }}$ week after vaccination, was significantly differed $(p<0.05)$ for both Cobb and Ross breeds. The highest values were found for all treated groups of both Cobb and Ross breed in comparison to control group. These results in agreement with Talebi et al. (2008), Landy and Kavyani (2013) and Shahir et al. (2014) who found that probiotic improve the antibody responses to ND. also agreed with Oliveira et al. (2009) who found that antibody titer against New castle increase in third week post vaccination in diet supplemented with MOS. also our results agreed with Dehghani and Jahanian (2012) who found that antibody titers against Newcastle at day 12 post vaccine inoculation was significantly $(P<0.01)$ affected by organic acid addition. Regarding antibody titer results for enzyme supplemented group, it was in agreement with Soltan (2009) who mentioned that enzyme had no effect $(P>0.05)$ on HI titer of broiler chicks when compared with the chicks fed on the same diet without enzyme supplementation.

Similar results were obtained by Houshmand et al. (2012) who found that antibody titer to Newcastle disease virus was higher in all treated group (probiotic, prebiotic and organic acid) compared to control group, which indicated positive effect of treated group on immunity. Also Amal, et al. (2013) reported that Newcastle disease (ND) vaccination for the Probiotic and Prebiotics supplemented birds was significantly improved in comparison with the vaccinated non treated control group. From the previous results, we found that all diet supplementation had appositive effect on antibody titer to vaccination against Newcastle vaccine.

Regarding albumin value table (7), the highest value was found for Cobb breed treated with probiotic supplementation (2.30). The globulin showed higher value for all treated groups in comparison to control group except for probiotic treated group of Cobb breed. The Value of total protein for Cobb breed was high in all treated groups in comparison to control group. Value of albumin to globulin ratio was low in all treated group of Cobb breed except for probiotic treated group (probiotic had an increased value of albumin in comparison to globulin).

These results agreed with Ashayerizadeh et al. (2009) who found that total protein was high in treated group (probiotic and prebiotic treated) in compared to control group. Also, Abdel Raheem and Abd Allah (2011) stated that total protein was high in treated group (probiotic, prebiotic and synbiotic treated) compared to control group. High value in organic acid treated was agreed with Azza and Naela (2014) who indicated positive effect of organic acid on value of total protein. While in Ross breed showed the lowest value in diet supplemented with prebiotic treated group. similar results reported by Shahir et al. (2014).

Concerning results in table (8), they revealed that the logarithmic production function was significant $(P<0.05)$, and about $70 \%$ from the changes in body weight were attributed to changes in production resources. As shown from the results, the average elasticity of production resources (feed cost, additive cost and drug cost) was about $(+6.38)$, meaning that increasing this resources by $1 \%$ resulted in increase of body weight by $(6.38 \%)$. the average elasticity of feed cost was about $(+0.38)$, meaning that increasing feed cost by about $10 \%$ resulted in increase of body weight by $(3.8 \%)$. Our results was in agreement with Willems et al. (2013) who found that positive relationship between feed intake and body weight of broiler chicken. The average elasticity of drug cost was about (+5.96); meaning that increasing drug cost by about $1 \%$ resulted in increase of body weight by $(5.96 \%)$.

The average elasticity of feed additive cost was about $(+0.044)$, meaning that increasing feed additive cost by about $10 \%$ resulted in increase of body weight by $(0.4 \%)$, which was in agreement with Hassanein (2006), Zhang et al. (2006), Nayebpor et al. (2007), Abdel Fattah e al. (2008), Adil et al., 2010, Azza and Naela (2014) and ElFaham et al. (2015), who reported that broiler chicks of dietary feed additive supplementation improves body weight. Table (9), clarifies that the average elasticity of feed additive cost was about $(+0.001)$, meaning that increasing feed additive cost by about $10 \%$ resulted in increase of total cost by $(0.01 \%)$. As feed additive is a part of the cost used in the production process. Table (10), shows that the average elasticity of feed additive cost was 
about (+0.067), meaning that increasing feed additive cost by about $10 \%$ resulted in increase of total return by $(0.67 \%)$.

\section{REFERENCES}

Abdel Fattah, S.A., El-Sanhoury, M.H., ElMednay, N.M. and Abdel Azeem, F. 2008. Thyroid Activity, Some Blood Constituents, Organs Morphology and Performance of Broiler Chicks Fed Supplemental Organic Acids International Journal of Poultry Science 7 (3): 215-222.

Abdel Raheem, S.M. and Abd-Allah, S.M.S. 2011. The Effect of Single or Combined Dietary Supplementation of Mannan Oligosacharide and Probiotics on Performance and Slaughter Characteristics of Broilers. International Journal of Poultry Science 10 (11): 854-862.

Adil, S., Banday, B., Bhat, G.A., Mir, M.S. and Rehman, M. 2010. Effect of Dietary Supplementation of Organic Acids on Performance, Intestinal Histomorphology, and Serum Biochemistry of Broiler Chicken. SAGE-Hindawi Access to Research Veterinary Medicine International, Article ID 479485, 7 pages.

Afifi, E.M. 1988. Analytical study for broiler production in Egypt. M.V.Sc. Thesis, Fac. of Agri., Cairo University.

Al Saad, S., Abbod, M. and Abo Yones, A. 2014. Effects of some Growth Promoters on Blood Hematology and Serum Composition of Broiler Chickens International Journal of Agricultural Research, 9: 265-270.

Amaechi, N. and Amaeze, P.N. 2012. Effect of dietary chloroacetic acid as antibiotic replacer on the gastrointestinal micro flora and gut morphology of weanling pigs. Res. Opin. Anim. Vet. Sci 2: 494-498.

Amal, H. T. Abdelnaser, Suzan, H. Tolba and Marwa, I. Abd El-Haleem . 2013. Effect of using biotic products as alternatives to antibiotics in broiler chickens Benha Veterinary Medical Journal 24 (2): 44-57.

Amer, A.M. 2014. Effect of phytase enzyme supplementation in deficient phosphate diet on broiler performance and some blood. International Journal of Science and Nature 5 (2): $341-345$

Ashayerizadeh, O., Dastar, B. ,Shams Shargh, M., Ashayerizadeh, A. and Mamooee, M. 2009. Influence of Antibiotic, Prebiotic and Probiotic Supplementation to Diets on
Carcass Characteristics, Hematological Indices and Internal Organ Size of Young Broiler Chickens. Journal of Animal and Veterinary Advances 8 (9): 1772-1776.

Atallah, S.T. 1994. Economic and productive efficiency of veterinary therapy in poultry farms. M.V.Sc. Thesis, Fac. Vet. Med., Alex. Univ., Egypt.

Atallah, S.T. 1997: Economic and productive efficiency of veterinary management in dairy farms. Ph.D. Thesis, Fac. Vet. Med., Alex. Univ., Egypt.

Azza, M. Kamal and Naela, M. Ragaa 2014. Effect of Dietary Supplementation of Organic Acids on Performance and Serum Biochemistry of Broiler Chicken. Nature and Science 12(2):38-45.

Beski, S.S.M. and Al Sardary, S.Y.T. 2015. Effects of Dietary Supplementation of Probiotic and Synbiotic on Broiler Chickens Hematology and Intestinal Integrity. International Journal of Poultry Science 14 (1): 31-36.

Bozkurt, M., Küçükyılmaz, K., Çatlı, A.U. and Çınar, M. 2009. The effect of single or combined dietary supplementation of prebiotics, organic acid and probiotics on performance and slaughter characteristics of broilers. South African Journal of Animal Science 39 (3):197-205.

Campbell, T. W.1995. Avian hematology and cytology. Iowa State University Press

Chen, Y.C., Nakthong, C. and Chen, T. C. 2005. Improvement of laying hen performance by dietary prebiotic chicory oligofructose and inulin, Int. J. Poult. Sci 4. 170-178.

Dehghani, N. and Jahanian, R. 2012. Effects of dietary organic acid supplementation on immune responses and some blood parameters of broilers fed diets with different protein levels, World's Poult. Sci. J. 68:S1S4.

Doll, J.P. and Orazem, F. 1978. Production economics theory with applications. Grid Inc. Columbus, Ohio, USA.

Duncan, D. B.1955. Multiple range and multiple F tests. Biometrics 11:1-42.

El Faham, A.I., Nematallah G.M. Ali and Amal M. Hassan. 2015. Productive Performance and Blood Plasma Constituents of Broiler chicks fed diets supplemented with some natural feed additives. Egyptian J. Nutrition and Feeds 18(2): 153-162.

Fardos, A. Hassan. 2009. Effect of Some Feed Additives on Economic and Productive Efficiency of Broilers. Master degree, faculty 
of veterinary medicine, Zagazig University Egypt.

Gao, J., Zhang, H.J., Yu, S.H., Wu, S.G., Yoon, I., Quigley, J., Gao, Y.P. and Qi, G.H. 2008. Effects of yeast culture in broiler diets on performance and immunomodulatory functions. Poult .Sci. 87:1377-1384.

Hassanein, H.H. 2006. Effect of dietary Alpha Yea Mix supplementation on boiler chickens performance. Egypt Poult. Sci. 26:12851303.

Haque M. N., Islam, K. M. S., Akbar, M. A., Chowdhury, R., Khatun, M., Karim, M. R. and Kemppainen B. W. 2010.Effect of dietary citric acid, flavomycin and their combination on the performance, tibia ash and immune status of broiler. Can. J. Anim. Sci. 90: 57-63.

Houshmand, M., Azhar, K., Zulkifli,I., Bejo, M.H. and Kamyab, A. 2012. Effects of nonantibiotic feed additives on performance, immunity and intestinal morphology of broilers fed different levels of protein. South African. J. Anim. Sci. 42 (1):22-32.

Islam, M.S., Lucky N.S., Islam, M.R., Ahad, A., Das, B.R., Rahman M.M. and Siddiui, M.S.I. 2004. Haematological parameters of Fayoumi, Assil and local chickens reared in Sylhet region in Bangladesh. Int. J. Poult. Sci. 3: 144-147.

Kamruzzaman, S.M., Kabir, S.M.L., Rahman, M.M. Islam, M.W. and Reza, M.A. 2005. Effect of probiotics and antibiotics supplementation on body weight and certain haemato-biochemical parameters in broilers .Bangl. J. Vet. Med. 3(2):100-104.

Khan, R.U., Naz, S. and Dhama, K., 2014. Chromium: pharmacological applications in heat stressed poultry. Int. J. Pharmacol 10: 213-317.

Khosravi, A., Boldaji, F., Dastar, B. and Hasani, S .2010. Immune Response and Performance of Broiler Chicks Fed Protexin and Propionic Acid. International. J. Poult. Sci. 9 (2): 188191.

Landy, N. and Kavyani, A. 2013. Effects of Using a Multi-Strain Probiotic on Performance, Immune Responses and Cecal Microflora Composition in Broiler Chickens Reared Under Cyclic Heat Stress Condition. Iranian Journal of Applied Animal Science. 3 (4): 703-708.

Mahdavi, R. and Torki, M. 2009. Study on usage period of dietary protected butyric acid on performance, carcass characteristics, serum metabolite levels and humoral immune response of broiler chickens. Journal of Animal and Veterinary Advances 8(9):17021709.

Majiyagble, L.A. and Hitchner, S.B. 1977. Antibody response to strain combination of Newcastle disease virus as measured by hemagglutination inhibition. Avain diseases 21: 576-584.

Marwa, I. Abdel-Haleem 2013. Use of Biotic Preparations as an Alternative to Antibiotics in Broiler Chickens Under Semifield and Field Conditions. Master Degree in Veterinary Medicine Science (Poultry Diseases) Benha University.

National Research Council (NRC) 1994. Nutrient Requirements of poultry $.9^{\text {th }} \mathrm{Ed}$, Washington, DC National academy press.

Nayebpor, M., Farhomand, P. and Hashemi, A. 2007. Effect of different levels of direct fed microbial (Primalac) on the growth performance and humoral immune response in broiler chickens. J. Anim. Adv 6: 13081313.

Nikpiran, H., Taghavi, M., Khodadadi, A. and Athari, S. S. 2013.Influence of Probiotic and Prebiotic on broiler chickens performance and immune status. Journal of Novel Applied Sciences. 2(8):256-259

Oliveira, M.C., Figueiredo-Lima, D.F., Faria Filho, D.E., Marques R.H., and Moraes, V. M. B. 2009. Effect of mannanoligosaccharides and/or enzymes on antibody titers against infectious bursal and Newcastle disease viruses. Arq. Bras. Med. Vet. Zootec., 61(1): 6-11.

Owosibo A. O., Odetola, O. M., Odunsi ,O. O. and Lawrence-Azua, O. O. 2013. Growth, hematology and serum biochemistry of broilers fed probiotics based diets African Journal of Water Conservation and Sustainability1 (4): 61-65.

Roser, U. 2006. Effects of organic acids in liquid and solid forms on the survival rate of Salmonella in pelleted compound feed after recontamination, J. Immunol. 82 : 9-12.

Saffari Samani, E., Zare Shahneh A., Pourreza, J. and Vali, N. 2012. The effect of supplementation probiotic BIOSAF on the immune response to Newcastle vaccination and SRBC injection.3rd international poultry congress. Tehran-Iran.

Saiyed, M. A., Joshi, R. S., Savaliya, F. P., Patel, A. B., Mishra, R. K. and Bhagora, N. J. 2015. Study on inclusion of probiotic, prebiotic and 
its combination in broiler diet and their effect on carcass characteristics and economics of commercial broilers. Veterinary World 8(2): 225-231.

Salim, H.M., Kang, H.K., Akter, N., Kim, D.W., Kim, J.H., Kim, M.J., Na, J.C., Jong, H.B., Choi, H.C., Suh, O.S. and Kim, W.K. 2012. Supplementation of direct-fed microbials as an alternative to antibiotic on growth performance, immune response, cecal microbial population, and ileal morphology of broiler chickens. Poult. Sci. 92(8):208490.

Shahir, M.H., Afsarian, O., Ghasemi, S. and Tellez, G. 2014. Effects of Dietary Inclusion of Probiotic or Prebiotic on Growth Performance, Organ Weight, Blood Parameters and Antibody Titers Against Influenza and Newcastle in Broiler Chickens. International. J. Poult. Sci. 13 (2): 70-75.

Soltan, M. A. 2009. Growth performance, immune response and carcass traits of broiler chicks fed on graded levels of palm kernel cake without or with enzyme supplementation live stock Research for Rural Development. 21 (3).

SPSS 2004. Statistical Package for Social Sciences. Release 16.0.1 version. SPSS Inc.
Stoot, G. H. and Fellah, A. 1983. Colostral immunoglobulin absorption nearly related to concentration for calves. J. dairy. Sci. 66: 1315-1328.

Talebi, A., Amirzadeh, B., Mokhtari B. and Gahri, H. 2008. Effects of a multi-strain probiotic (Prima Lac) on performance and antibody responses to Newcastle disease virus and infectious bursal disease virus vaccination in broiler chickens. Avian Pathol. 37(5): 509512.

Talebi, A., Asri-Rezaei, S., Rozeh-Chai, R. and Sahraei, R. 2005. Comparative Studies on Haematological Values of Broiler Strains (Ross, Cobb, Arbor-acres and Arian. International. J. Poult. Sci. 4 (8): 573-579.

Willems, O.W., Miller, S.P. and Wood. B.J. 2013. Assessment of residual body weight gain and residual intake and body weight gain as feed efficiency traits in the turkey (Meleagris gallopavo). Genet Sel Evol. 45(1):26. Published online 2013 Jul 16. doi: $10.1186 / 1297-9686-45-26$

Zhang, G., Ma, L. and Doyle, M.P. 2006. Efficiency of probiotics, prebiotics and synbiotics on weight increase of chickens (Gallus Domesticus); Retrieved from: http:// www.ugacfs.org/research/pdfs/Poultry2006. pdf. 